\title{
我国玉米自交系叶片保绿性及其与产量的关系
}

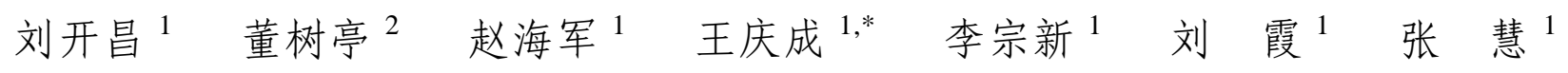

${ }^{1}$ 山东省农业科学院玉米研究所, 山东济南 $250100 ;{ }^{2}$ 山东农业大学作物生物学国家重点实验室, 山东泰安 271018

摘 要: 选用我国 75 个常用不同基因型玉米自交系, 对其叶片保绿性参数进行了定点动态测定。结果表明, 不同自 交系抽丝后叶片保绿度的变化均符合方程 $y=a \mathrm{e}^{b-c x} /\left(1+\mathrm{e}^{b-c x}\right)$, 成熟期的绿叶数、成熟期叶绿素含量和相对绿叶面积 平均衰减速率 $\left(V_{\mathrm{m}}\right)$ 可作为区分玉米保绿型与非保绿型的关键指标。按照 Hiechical 聚类分析方法, 篮选出 12 个保绿型 自交系, 其成熟期相对绿叶面积在 $60 \%$ 以上, 其 $V_{\mathrm{m}}$ 平均值为 $0.687 \% \mathrm{~d}^{-1}$, 在生长季内相对绿叶面积无大幅度衰减, 成 熟期绿叶数多, 叶绿素含量较高; 其余 63 个为非保绿型自交系, 还可分为植株叶片衰老较快型与植株叶片衰老较慢 型两个亚类。不同自交系抽丝后叶片保绿性与叶面积持续期、单株产量均呈正相关。保绿型的叶面积持续期和单株 产量比非保绿型分别高 $20.02 \%$ 23.87\%和 $50.44 \%$ 59.38\%; 与非保绿型自交系相比, 保绿型在籽粒灌浆期绿叶面积 大, 叶绿素含量高, 群体光合速率高, 光合作用时间长, 因而生物产量较高。

关键词: 玉米; 自交系; 保绿性; 产量

\section{Leaf Stay-Green Traits in Chinese Maize Inbred Lines and Their Relationship with Grain Yield}

\author{
LIU Kai-Chang ${ }^{1}$, DONG Shu-Ting ${ }^{2}$, ZHAO Hai-Jun ${ }^{1}$, WANG Qing-Cheng ${ }^{1, *}$, LI Zong-Xin ${ }^{1}$, LIU Xia ${ }^{1}$, and \\ ZHANG Hui $^{1}$ \\ ${ }^{1}$ Maize Research Institute, Shandong Academy of Agricultural Sciences, Jinan 250100, China; ${ }^{2}$ State Key Laboratory of Crop Biology, Shandong \\ Agricultural University, Tai’an 271018, China
}

\begin{abstract}
Leaf stay-green trait is one of the major targets in maize (Zea mays L.) breeding. Although sugar concentration in culm, green leaf number, leaf area, and chlorophyll content at maturity have been used to evaluate the stay-green trait of maize in earlier studies, there is no a common criterion. To further study the evaluation system for stay-green in maize and disclose the relationship of stay-green with grain yield, we sampled 75 inbred lines from common parents of Chinese maize cultivars. At silking stage and 10, 20, 30, 40, 50, and $60 \mathrm{~d}$ after silking, the leaf area (LA), leaf area duration (LAD), relative leaf area, and relative green-leaf area (RGLA) were measured. The results showed that the changes of RGLA after silking accorded with the equation of $y=a \mathrm{e}^{b-c x} /\left(1+\mathrm{e}^{b-c x}\right)$. On the basis of correlation analysis, the green leaf number, chlorophyll content at physiological maturity, and mean decreasing rate of RGLA $\left(V_{\mathrm{m}}\right)$ were selected as the key indices to discriminate the stay-green and non-stay-green maize genotypes. According to relative green leaf area at physiological maturity (MRGLA), the maximum decrease rate of RGLA ( $\left.V_{\max }\right)$, and the mean decreasing rate of RGLA $\left(V_{\mathrm{m}}\right)$, the 75 inbred lines were classified into stay-green and non-stay-green two types with Hiechical clustering analysis. The stay-green type was composed of 12 inbred lines with the following common characteristics: MRGLA of more than $60 \%, V_{\mathrm{m}}$ of $0.687 \% \mathrm{~d}^{-1}$, no significant decrease of RGLA during the whole growing period (67.07\% at maturity), and high green leaf number (8.8 leaves) and chlorophyll content (4.43 $\mathrm{mg} \mathrm{dm}^{-2}$ ) at physiological maturity. The non-stay-green type, consisting of 63 inbred lines, was further categorized with quick-leaf-senescence (50 inbred lines) and slow-leaf-senescence (13 inbred lines) subgroups. In the quick- and slow-leaf senescence subgroups, the RGLA at maturity, $V_{\mathrm{m}}$, green leaf number at maturity, and chlorophyll content at maturity were $17.75 \%$ and $33.55 \%, 1.89 \% \mathrm{~d}^{-1}$ and $1.44 \% \mathrm{~d}^{-1}, 3.2$ and 6.2, and $2.06 \mathrm{mg} \mathrm{dm}^{-2}$ and $3.17 \mathrm{mg} \mathrm{dm}^{-2}$, respectively. At physiological maturity, the RGLA was positively correlated with LAD $(r=0.8861, P<0.01)$ and yield per plant $(r=0.8221, P<0.01)$. The LAD and yield per plant were $20.02-23.87 \%$ and $50.44-59.38 \%$ higher in the stay-green type than in the non-stay-green type, respectively. Thus, the stay-green type had higher
\end{abstract}

\footnotetext{
本研究由国家 十一五”科技支撑计划项目(2006BAD02A09, 2007BAD89B09, 2007BAD31B03)，山东省农业科学院博士基金项目(2007YBS002), 山东省农业科学院高技术自主创新基金(2007YCX024)资助。

*通讯作者(Corresponding author): 王庆成, E-mail: qcwang@saas.ac.cn; Tel: 0531-83179402

第一作者联系方式: E-mail: liukc@saas.ac.cn

Received(收稿日期): 2008-12-31; Accepted(接受日期): 2009-04-23.
} 
yield potential due to larger green leaf area, higher chlorophyll content and photosynthesis efficiency as well as longer photosynthesis duration.

Keywords: Maize; Inbred line; Stay-green; Yield

叶片衰老外形上主要表现为叶色由绿变黄直至 全叶枯黄, 其典型特征是叶绿素降解和光合能力降 低 ${ }^{[1]}$ 。保绿性是指植株在籽粒生理成熟期叶片因衰 老进程延缓而无明显失绿, 是育种选择的理想性状 [2-9]。保绿性强的作物对病虫害和倒伏抗性较强 ${ }^{[10]}$, 其生物产量、籽粒产量和秸秆营养价值也较高 ${ }^{[11-12]}$, 并且便于机械化收割 ${ }^{[13]}$ 。玉米是我国重要的粮食和 饲料作物, 选育高产、多抗、广适性的青饲或粮饲 兼用玉米品种是目前育种的主攻方向之一 ${ }^{[14]}$, 保绿 型被认为是玉米品种的演进方向 ${ }^{[15-17]}$ 。关于保绿型玉 米的界定尚无统一标准, 通常以茎秆糖浓度 ${ }^{[18-19]}$ 、籽 粒生理成熟期的绿叶数 ${ }^{[2,20-21]}$ 、叶面积 ${ }^{[22-23]}$ 和叶绿素 含量 ${ }^{[2,10,24]}$ 等作为评价指标。国内关于玉米保绿性的 研究尚处于起步阶段 ${ }^{[25-27]}$, 进一步建立完善科学有 效的玉米保绿性评价指标体系, 探讨玉米保绿性的 生物特征及其生理机制, 具有重要的理论意义和应 用价值。本文旨在确立科学的玉米保绿性指标评价 体系, 为保绿型玉米新品种选育提供理论依据。

\section{1 材料与方法}

\section{1 试验设计}

2002-2005 年, 在山东省济南市山东省农业科 学院试验农场种植我国玉米育种常用的 75 个自交系 (表 1), 6 月 10 日播种, 9 月 17 日收获。试验田地力 均匀, 耕作层内含有机质 $2.26 \%$, 碱解氮 $75.34 \mathrm{mg}$ $\mathrm{kg}^{-1}$, 速效磷 $29.28 \mathrm{mg} \mathrm{kg}^{-1}$, 速效钾 $218.57 \mathrm{mg} \mathrm{kg}^{-1}$, $\mathrm{pH}$ 7.53。每材料设 3 行区, 行距 $66.6 \mathrm{~cm}$, 行长 $5 \mathrm{~m}$, 3 次重复, 完全随机排列。种植密度为 6 万株 $\mathrm{hm}^{-2}$ 。 施肥、浇水等田间管理措施同常规大田生产。拔节 期, 每个自交系 1 行内连续选取 10 株, 用红漆标记, 用于定点观测 ${ }^{[14]}$ 。

\section{2 测定指标和方法}

于抽丝期和抽丝后 $10 、 20 、 30 、 40 、 50$ 和 $60 \mathrm{~d}$ 调查单株叶面积和绿叶数。单叶叶面积 $(L A)=L \times W \times$ $0.75(L \text { 表示叶片中脉长度, } W \text { 表示叶片最大宽度 })^{[14]}$; 叶面积持续期 $(L A D)=\left(L A_{2}+L A_{1}\right) \times\left(T_{2}-T_{1}\right) / 2\left(L A_{1}\right.$ 和 $T_{1}$ 分别表示前一次的叶面积、取样时间, $L A_{2}$ 和 $T_{2}$ 分别 表示后一次的叶面积、取样时间 ${ }^{[14]}$; 相对绿叶面积 $($ RGLA $)=$ 抽丝后某时刻绿叶面积/抽丝期最大绿叶
面积 $\times 100 \%$ (以玉米抽丝期绿叶面积为 $100 \%$ ); 以生 理成熟期的相对绿叶面积(\%)表示保绿度(stay-green degree)。相对绿叶面积变化特征用曲线方程 $y=$ $a \mathrm{e}^{b-c x} /\left(1+\mathrm{e}^{b-c x}\right)$ 描述, 其中, $y$ 为某一时刻的 RGLA (\%), $x$ 为抽丝后的天数, 参数 $a$ 为 RGLA 的理论初始 值, $b$ 与叶片衰老的启动有关, $c$ 与叶片衰老的速度有 关 ${ }^{[29]}$ 。对 75 个自交系 RGLA的变化曲线方程求导, 得 到不同基因型间相对绿叶面积最大衰减速率 $\left(V_{\max }\right)$ 和出现最大绿叶衰减速率的时间 $\left(T_{\max }\right)$ 。定义植株相 对绿叶面积达到 $95 \%$ 时为衰老的启动时间 $\left(T_{\mathrm{s}}\right)$ 。

用 Arnon 法 ${ }^{[28]}$ 测定玉米抽丝期和生理成熟期叶 绿素含量, 用 UV-160A 分光光度计比色。成熟期每 个重复各收获 1 行, 按常规方法测产。

\section{3 数据分析}

采用 Hiechical 法进行聚类分析, 用 Microsoft Excel 和 SPSS 软件进行数据处理、显著性分析与相 关性分析。

\section{2 结果与分析}

2.1 不同玉米基因型叶片保绿性的动态变化 75 个自交系抽丝后相对绿叶面积的衰减符合“慢 一快一慢”的趋势(图 1)。

不同自交系抽丝后 RGLA 的变化特征由表 2 所 示性状参数来描述。不同自交系玉米成熟期 RGLA 的变化幅度为 $9.91 \% ~ 71.26 \%$, 平均值为 $28.45 \%$, 其 标准偏差为 18.87, 差异达显著水平 $\left(q_{0.01,75}=5.79\right)$; 不同基因型间 $V_{\text {max }}$ 与 $T_{\text {max }}$ 的标准方差分别为 5.98、 11.65, 差异均达显著水平。 $T_{\mathrm{s}}$ 的变化范围是 10.06 $13.74 \mathrm{~d}$, 最高相差 $3.68 \mathrm{~d}$, 其标准偏差为 0.76 , 差异 不显著 $\left(q_{0.05,75}=4.95\right)$ 。

2.2 不同基因型玉米自交系叶片保绿性的聚类 分析与划分

依据成熟期相对绿叶面积和叶片衰减速率, 按 照 Hiechical 聚类分析方法, 将供试玉米自交系分为 保绿型和非保绿型两类(图 2)。其中, 保绿型包括 12 个自交系，分别是 9706、NX531、齐 319、齐 318、 178、137、VA91、KW1、CZ01、97-21、7381、3195, 其成熟期 RGLA 均值为 67.07\% (60.07\% 71.26\%); $V_{\mathrm{m}}$ 均值为 $0.687 \% \mathrm{~d}^{-1}\left(0.516 \sim 0.868 \% \mathrm{~d}^{-1}\right), V_{\text {max }}$ 均值为 
表 1 不同玉米自交系抽丝后相对绿叶面积的动态变化

Table 1 Dynamic changes of relative green leaf area (RGLA) of maize inbred lines after silking

\begin{tabular}{|c|c|c|c|c|c|c|c|c|c|c|c|}
\hline \multirow{2}{*}{ No. } & \multirow{2}{*}{$\begin{array}{c}\text { 自交系 } \\
\text { Inbred line }\end{array}$} & \multicolumn{3}{|c|}{ 方程参数 Parameter ${ }^{1)}$} & \multirow{2}{*}{$\begin{array}{c}\text { 相关系数 } \\
r \\
\end{array}$} & \multirow{2}{*}{ No. } & \multirow{2}{*}{$\begin{array}{c}\text { 自交系 } \\
\text { Inbred line }\end{array}$} & \multicolumn{3}{|c|}{ 方程参数 Parameter ${ }^{1)}$} & \multirow{2}{*}{$\begin{array}{c}\text { 相关系数 } \\
r\end{array}$} \\
\hline & & $a$ & $b$ & $c$ & & & & $a$ & $b$ & c & \\
\hline 1 & 齐 319 Qi 319 & 103.6 & 3.51 & -0.045 & $0.9957^{* *}$ & 39 & 黄早四 Huangzao 4 & 100.9 & 4.23 & -0.107 & $0.9924^{* *}$ \\
\hline 2 & 齐 318 Qi 318 & 103.6 & 3.48 & -0.044 & $0.9910^{* *}$ & 40 & 丹 340 Dan 340 & 101.4 & 4.08 & -0.084 & $0.9907^{* *}$ \\
\hline 3 & 武 314 Wu 314 & 101.3 & 4.18 & -0.102 & $0.9902^{* *}$ & 41 & 9046 & 101.3 & 4.09 & -0.091 & $0.9960^{* *}$ \\
\hline 4 & LY92 & 101.7 & 3.58 & -0.047 & $0.9974^{* *}$ & 42 & 吉 853 Ji 853 & 101.7 & 3.58 & -0.047 & $0.9957^{* *}$ \\
\hline 5 & Mo17 & 101.4 & 4.29 & -0.113 & $0.9934^{* *}$ & 43 & 齐 302 Qi 302 & 101.4 & 4.13 & -0.106 & $0.9966^{* *}$ \\
\hline 6 & 178 & 102.9 & 3.57 & -0.049 & $0.9950^{* *}$ & 44 & $5 \mathrm{~m} 121$ & 101.7 & 3.92 & -0.097 & $0.9971^{* *}$ \\
\hline 7 & 137 & 102.9 & 3.57 & -0.047 & $0.9947^{* *}$ & 45 & 掖 502 Ye 502 & 101.5 & 4.09 & -0.098 & $0.9971^{* *}$ \\
\hline 8 & 478 & 101.9 & 3.87 & -0.077 & $0.9974^{* *}$ & 46 & ZP118 & 101.7 & 4.05 & -0.097 & $0.9970^{* *}$ \\
\hline 9 & $\mathrm{BM}$ & 101.3 & 4.15 & -0.107 & $0.9868^{* *}$ & 47 & 齐 201 Qi 201 & 101.1 & 4.19 & -0.100 & $0.9905^{* *}$ \\
\hline 10 & M51 & 101.5 & 3.99 & -0.099 & $0.9878^{* *}$ & 48 & 52106 & 101.6 & 3.98 & -0.096 & $0.9957^{* *}$ \\
\hline 11 & 8112 & 101.8 & 3.94 & -0.095 & $0.9982^{* *}$ & 49 & 5005 & 101.4 & 4.02 & -0.088 & $0.9847^{* *}$ \\
\hline 12 & NX531 & 102.8 & 3.60 & -0.055 & $0.9974^{* *}$ & 50 & 齐 205 Qi 205 & 101.5 & 3.93 & -0.093 & $0.9963^{* *}$ \\
\hline 13 & 757 & 101.4 & 4.07 & -0.099 & $0.9977^{* *}$ & 51 & 3141 & 101.1 & 4.26 & -0.107 & $0.9903^{* *}$ \\
\hline 14 & 653 & 101.8 & 4.04 & -0.097 & $0.9918^{* *}$ & 52 & CZ01 & 102.7 & 3.52 & -0.047 & $0.9970^{* *}$ \\
\hline 15 & 9333 & 101.6 & 4.11 & -0.090 & $0.9960^{* *}$ & 53 & $\mathrm{CZ02}$ & 102.3 & 3.84 & -0.085 & $0.9864^{* *}$ \\
\hline 16 & H99 & 101.7 & 4.02 & -0.087 & $0.9977^{* *}$ & 54 & 郑 58 Zheng 58 & 101.9 & 4.04 & -0.083 & $0.9917^{* *}$ \\
\hline 17 & B73 & 101.4 & 4.18 & -0.103 & $0.9971^{* *}$ & 55 & E28 & 101.8 & 4.04 & -0.085 & $0.9949^{* *}$ \\
\hline 18 & 吉 846 Ji 846 & 101.1 & 4.08 & -0.099 & $0.9864^{* *}$ & 56 & 黄 C Huang C & 101.6 & 3.96 & -0.083 & $0.9943^{* *}$ \\
\hline 19 & 郑 32 Zheng 32 & 101.2 & 4.11 & -0.099 & $0.9893^{* *}$ & 57 & 昌 7-2 Chang 7-2 & 101.0 & 4.20 & -0.101 & $0.9914^{* *}$ \\
\hline 20 & 冀 35 Ji 35 & 101.0 & 4.28 & -0.103 & $0.9919^{* *}$ & 58 & 掖 515 Ye 515 & 101.3 & 4.10 & -0.096 & $0.9939^{* *}$ \\
\hline 21 & 9706 & 102.7 & 3.62 & -0.054 & $0.9953^{* *}$ & 59 & 自 330 Zi 330 & 101.2 & 4.19 & -0.101 & $0.9939^{* *}$ \\
\hline 22 & 凤直 Fengzhi & 101.7 & 4.35 & -0.109 & $0.9869^{* *}$ & 60 & 9331 & 101.3 & 4.21 & -0.097 & $0.9928^{* *}$ \\
\hline 23 & KH5 & 101.4 & 4.25 & -0.106 & $0.9990^{* *}$ & 61 & $97-21$ & 102.7 & 3.55 & -0.049 & $0.9993^{* *}$ \\
\hline 24 & 金 54 Jin 54 & 100.9 & 4.17 & -0.108 & $0.9792^{* *}$ & 62 & 齐 401 Qi 401 & 101.2 & 4.22 & -0.102 & $0.9955^{* *}$ \\
\hline 25 & VA-91 & 103.3 & 3.54 & -0.048 & $0.9944^{* *}$ & 63 & Wx303 & 101.2 & 4.13 & -0.096 & $0.9914^{* *}$ \\
\hline 26 & 冀 815 Ji 815 & 101.8 & 3.86 & -0.079 & $0.9936^{* *}$ & 64 & WH3143 & 101.4 & 4.01 & -0.093 & $0.9920^{* *}$ \\
\hline 27 & 338 & 101.2 & 4.24 & -0.105 & $0.9949^{* *}$ & 65 & $\mathrm{H} 21$ & 101.2 & 4.04 & -0.095 & $0.9896^{* *}$ \\
\hline 28 & 200B & 100.9 & 4.23 & -0.108 & $0.9885^{* *}$ & 66 & 京 7 黄 Jing 7 Huang & 100.8 & 4.23 & -0.112 & $0.9774^{* * *}$ \\
\hline 29 & 5003 & 101.5 & 3.86 & -0.082 & $0.9862^{* *}$ & 67 & KW1 & 101.8 & 3.70 & -0.055 & $0.9712^{* *}$ \\
\hline 30 & Q1982 & 100.9 & 4.25 & -0.112 & $0.9882^{* *}$ & 68 & K1077 & 101.0 & 4.20 & -0.105 & $0.9911^{* *}$ \\
\hline 31 & 沈 118 Shen 118 & 101.3 & 4.10 & -0.094 & $0.9928^{* *}$ & 69 & 掖 488 Ye 488 & 101.9 & 3.73 & -0.061 & $0.9957^{* *}$ \\
\hline 32 & 7381 & 103.2 & 3.62 & -0.052 & $0.9900^{* *}$ & 70 & YW02 & 100.7 & 4.20 & -0.109 & $0.9693^{* *}$ \\
\hline 33 & 3195 & 103.1 & 3.59 & -0.050 & $0.9956^{* *}$ & 71 & 双 741 Shuang 741 & 101.0 & 4.18 & -0.106 & $0.9838^{* *}$ \\
\hline 34 & L9801 & 101.3 & 3.92 & -0.082 & $0.9648^{* *}$ & 72 & YM121 & 101.4 & 4.08 & -0.101 & $0.9933^{* *}$ \\
\hline 35 & 196 & 101.2 & 4.02 & -0.088 & $0.9889^{* *}$ & 73 & 7137 & 100.8 & 4.23 & -0.110 & $0.9851^{* *}$ \\
\hline 36 & 3189 & 101.7 & 3.94 & -0.078 & $0.9938^{* *}$ & 74 & WA141 & 101.6 & 3.94 & -0.094 & $0.9969^{* *}$ \\
\hline 37 & JNHZ4 & 101.4 & 4.04 & -0.091 & $0.9956^{* *}$ & 75 & AY311 & 101.4 & 4.06 & -0.098 & $0.9936^{* *}$ \\
\hline 38 & 掖 107 Ye 107 & 101.9 & 3.91 & -0.077 & $0.9972^{* *}$ & & & & & & \\
\hline
\end{tabular}

1) $a, b$, and $c$ are parameters of equation $y=a \mathrm{e}^{b-c x} /\left(1+\mathrm{e}^{b-c x}\right)$. ${ }^{* *}$ Significantly different at $P<0.01$. 


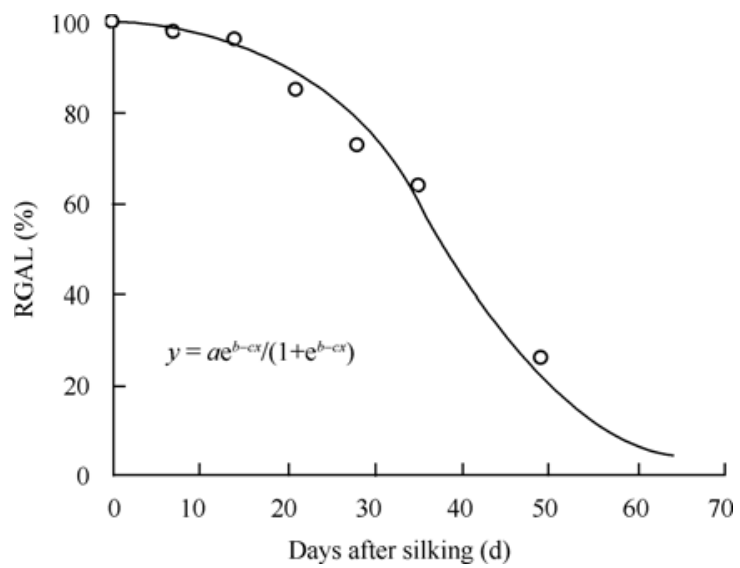

图 1 玉米抽丝后相对绿叶面积的动态变化

Fig. 1 Dynamic changes of relative green leaf area (RGLA) of maize after silking

$2.55 \% \mathrm{~d}^{-1}\left(2.26 \sim 2.81 \% \mathrm{~d}^{-1}\right), T_{\max }$ 的变幅为 $65.66 \sim$ $79.56 \mathrm{~d}$, 表明生长期内没有出现 $V_{\text {max。 }}$ 保绿型玉米 成熟期有绿叶 8 9 片, 平均 8.8 片, 成熟期果穗叶片 叶绿素含量为 $4.12 \sim 4.88 \mathrm{mg} \mathrm{dm}{ }^{-2}$, 平均 $4.43 \mathrm{mg}$ $\mathrm{dm}^{-2}$ (表 3)。

非保绿型包括 63 个自交系，其成熟期 RGLA 均 值为 $20.56 \%$ (9.91\% 42.33\%); $V_{\mathrm{m}}$ 均值为 $1.799 \% \mathrm{~d}^{-1}$ $\left(0.798 \sim 2.371 \% \mathrm{~d}^{-1}\right) ; V_{\max }$ 均值为 $4.862 \% \mathrm{~d}^{-1}(3.101 \sim$ $\left.5.719 \% \mathrm{~d}^{-1}\right) ; T_{\max }$ 的变幅为 $37.82 \sim 64.29 \mathrm{~d}$, 表明 $V_{\text {max }}$ 出现时间较早。非保绿型玉米自交系成熟期有绿叶 1 8 片不等, 平均比保绿型玉米自交系少 5.0 片, 成 熟期果穗叶片叶绿素含量范围是 1.28 3.80 $\mathrm{mg} \mathrm{dm}^{-2}$, 平均比保绿型玉米自交系小 $2.14 \mathrm{mg} \mathrm{dm}^{-2}$ 。

非保绿型自交系可分为叶片衰老较快型与衰老 较慢型两个亚类。衰老快型共 50 个自交系, 包括 K1077、自 330、Ym121、AY311、齐 201、CZ02、 757、653、M51、JNHZ4、9046、196、E28、9333、 H99、Wh3143、H21、Wx303、8112、齐 205、掖 515、9331、沈 118、BM、金 54、B73、Q1982、黄 早四、7137、凤直、KH5、338、3141、双 741、齐 302、京 7 黄、YW02、Mo17、掖 502、ZP118、52106、 吉 853、5m121、WA141、吉 846、郑 32、昌 7-2、 武 314、冀 35 和齐 401。其成熟期均值为 $17.75 \%$ (9.91\% 27.53\%), $V_{\mathrm{m}}$ 均值为 $1.89 \% \mathrm{~d}^{-1}(1.59 \sim 2.37 \%$ $\left.\mathrm{d}^{-1}\right), V_{\text {max }}$ 均值为 $5.06 \% \mathrm{~d}^{-1}\left(4.33 \sim 5.72 \% \mathrm{~d}^{-1}\right), T_{\text {max }}$ 的 变幅为 37.82 47.40 d。成熟期绿叶平均 3.2 片, 果穗 叶片叶绿素含量平均 $2.06 \mathrm{mg} \mathrm{dm}^{-2}$ (表 3)。

衰老慢型共 13 个自交系, 包括 478、3189、掖 107、5003、黄 C、丹 340、5005、LY92、L9801、 掖 488、郑 58、冀 815 和昌 7-2。其成熟期 RGLA 均
值为 $33.55 \%(27.80 \% ~ 42.33 \%), V_{\mathrm{m}}$ 均值为 $1.44 \% \mathrm{~d}^{-1}$ $\left(1.28 \sim 1.68 \% \mathrm{~d}^{-1}\right), V_{\max }$ 均值为 $4.06 \% \mathrm{~d}^{-1}(3.12 \sim 4.45 \%$ $\left.\mathrm{d}^{-1}\right), T_{\max }$ 的变幅为 $43.51 \sim 52.78 \mathrm{~d}$; 成熟期绿叶平均 6.2 片, 果穗叶片叶绿素含量平均 $3.17 \mathrm{mg} \mathrm{dm}^{-2}$ (表 3)。

叶片保绿性(相对绿叶面积)与成熟期的绿叶数、 成熟期叶绿素含量和最大速率时间 $\left(T_{\max (\mathrm{V})}\right)$ 呈极显 著正相关, 与抽丝期叶绿素含量呈显著正相关, 与 衰减启动时间 $\left(T_{95 \% \mathrm{RGLA}}\right)$ 和最大绿叶面积 $\left(L A_{\max }\right)$ 呈正 相关, 但未达到显著水平; 与平均衰减速率 $\left(V_{\mathrm{m}}\right)$ 呈 极显著负相关(表 4)。可以看出, 叶片的保绿主要取 决于成熟期的绿叶数、成熟期叶绿素含量和 $V_{\mathrm{m}}, V_{\mathrm{m}}$ 越大, 成熟期的绿叶数越少, 植株的保绿度越低。

\section{3 玉米叶片保绿性能与产量的相关性}

保绿型自交系 LAD 的变幅为 $15.04 \sim 24.18 \mathrm{~m}^{2} \mathrm{~d}$, 平均为 $19.26 \mathrm{~m}^{2} \mathrm{~d}$, 单株产量的变幅为 $68.70 \sim 143.40$ $\mathrm{g}$, 平均单株产量为 $101.28 \mathrm{~g}$; 非保绿型植株叶片衰 老较慢型 $\mathrm{LAD}$ 的变幅为 $10.01 \sim 17.41 \mathrm{~m}^{2} \mathrm{~d}$, 叶片衰 老较快型 $\mathrm{LAD}$ 的变幅为 $7.48 \sim 14.05 \mathrm{~m}^{2} \mathrm{~d}$, 二者平均 值分别比保绿型的低 $5.51 \mathrm{~m}^{2} \mathrm{~d}$ 和 $8.21 \mathrm{~m}^{2} \mathrm{~d}$; 二者的 单株产量的变幅范围也明显小于保绿型, 平均值分 别低 $30.50 \mathrm{~g}$ 和 $52.91 \mathrm{~g}$ 。方差分析表明, 保绿型自交 系的 LAD 和 YPP 与非保绿型、衰老较快型以及衰 老较慢型的差异均达到显著水平, 且衰老较快型的 $\mathrm{LAD}$ 和 YPP 与衰老较慢型的差异亦均达到显著水平, 但非保绿型自交系的 LAD 和 YPP 与其衰老较慢型 之间的差异不显著(表 5), 表明保绿型与非保绿型玉 米自交系存在本质上差异。

相关分析表明(图 3), 保绿度与 LAD 和单株产 量均呈极显著正相关, 其相关系数分别为 0.8861 和 $0.7812\left(r_{0.01,75}=0.302\right), \mathrm{LAD}$ 与单株产量亦呈极显著 正相关, 相关系数为 0.8221 。

\section{3 讨论}

同一作物的不同种质或基因型间叶片的保绿性 存在差异 ${ }^{[1]}$ 。国内外育种家和生理学家对作物保绿 性的评价标准已开展了许多研究 ${ }^{[9,30-34]}$ 。Xu 等 ${ }^{[10]}$ 和 Duncan 等 ${ }^{[13]}$ 认为叶绿素含量、绿叶面积、绿叶干重、 基部节间的含糖量等指标可作为划分高粱保绿性的 标准。在国外，叶片含水量、叶绿素含量、基部茎 秆的含糖量、绿叶片数、叶面积指数、叶面积持续 期和衰老的叶片数等也普遍作为区分玉米保绿型与

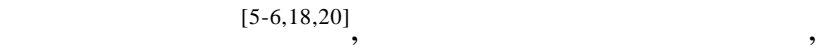
尚缺乏统一的鉴定标准 ${ }^{[25,27]}$ 。本研究表明, 不同基因 
表 2 不同自交系抽丝后相对绿叶面积性状参数的变化

Table 2 Changes of the trait parameters of RGLA after silking in different maize inbred lines

\begin{tabular}{|c|c|c|c|c|c|c|c|c|c|c|c|}
\hline $\begin{array}{c}\text { 自交系 } \\
\text { Inbred line }\end{array}$ & $\begin{array}{l}\text { MRGLA } \\
(\%)\end{array}$ & $\begin{array}{c}V_{\max } \\
\left(\% \mathrm{~d}^{-1}\right)\end{array}$ & $\begin{array}{c}V_{\mathrm{m}} \\
\left(\% \mathrm{~d}^{-1}\right)\end{array}$ & $\begin{array}{l}T_{\max } \\
\text { (d) }\end{array}$ & $\begin{array}{l}T_{\mathrm{s}} \\
\text { (d) }\end{array}$ & $\begin{array}{c}\text { 自交系 } \\
\text { Inbred line }\end{array}$ & $\begin{array}{l}\text { MRGLA } \\
(\%)\end{array}$ & $\begin{array}{c}V_{\max } \\
\left(\% \mathrm{~d}^{-1}\right)\end{array}$ & $\begin{array}{c}V_{\mathrm{m}} \\
\left(\% \mathrm{~d}^{-1}\right)\end{array}$ & $\begin{array}{l}T_{\max } \\
\text { (d) }\end{array}$ & $\begin{array}{l}T_{\mathrm{s}} \\
\text { (d) }\end{array}$ \\
\hline 齐 319 Qi 319 & 71.26 & 2.32 & 0.57 & 78.10 & 12.62 & 黄早四 Huangzao 4 & 12.01 & 5.41 & 2.05 & 39.43 & 11.97 \\
\hline 齐 318 Qi 318 & 70.79 & 2.26 & 0.58 & 79.57 & 12.15 & 丹 340 Dan 340 & 35.6 & 4.27 & 1.40 & 48.42 & 13.45 \\
\hline 武 314 Wu 314 & 18.3 & 5.15 & 1.99 & 41.17 & 12.19 & 9046 & 24.16 & 4.60 & 1.61 & 45.04 & 12.64 \\
\hline LY92 & 29.42 & 4.28 & 1.53 & 47.81 & 12.80 & 吉 853 Ji 853 & 15.47 & 4.95 & 1.88 & 41.94 & 11.80 \\
\hline Mo17 & 11.08 & 5.72 & 1.93 & 38.02 & 11.92 & |齐 302 Qi 302 & 14.86 & 5.20 & 1.89 & 40.28 & 11.56 \\
\hline 178 & 70.34 & 2.50 & 0.63 & 73.58 & 13.01 & $5 \mathrm{~m} 121$ & 15.78 & 4.94 & 1.91 & 40.40 & 10.06 \\
\hline 137 & 70.72 & 2.43 & 0.52 & 75.93 & 13.43 & 掖 502 Ye 502 & 16.59 & 4.98 & 1.81 & 41.69 & 11.69 \\
\hline 478 & 34.58 & 3.90 & 1.45 & 50.54 & 12.08 & ZP118 & 17.02 & 4.96 & 1.80 & 41.58 & 11.38 \\
\hline $\mathrm{BM}$ & 12.72 & 5.43 & 2.36 & 38.73 & 11.26 & |齐 201 Qi 201 & 20.82 & 5.05 & 1.76 & 41.92 & 12.45 \\
\hline M51 & 17.75 & 5.05 & 2.11 & 40.14 & 10.54 & 52106 & 17.11 & 4.90 & 1.84 & 41.26 & 10.71 \\
\hline 8112 & 17.53 & 4.81 & 1.92 & 41.62 & 10.48 & 5005 & 32.26 & 4.45 & 1.41 & 45.75 & 12.21 \\
\hline NX531 & 60.2 & 2.81 & 0.77 & 65.66 & 11.89 & |齐 205 Qi 205 & 18.26 & 4.72 & 1.74 & 42.30 & 10.62 \\
\hline 757 & 15.32 & 5.00 & 2.12 & 41.29 & 11.42 & 3141 & 15.25 & 5.40 & 1.93 & 39.81 & 12.28 \\
\hline 653 & 16.42 & 4.96 & 2.14 & 41.46 & 11.23 & |CZ01 & 70.91 & 2.39 & 0.65 & 75.75 & 12.44 \\
\hline 9333 & 25.44 & 4.55 & 1.86 & 45.85 & 12.98 & CZ02 & 15.49 & 4.33 & 1.88 & 45.49 & 10.68 \\
\hline H99 & 27.53 & 4.40 & 1.81 & 46.34 & 12.35 & 郑 58 Zheng 58 & 23.39 & 4.23 & 1.67 & 48.65 & 13.23 \\
\hline B73 & 13.64 & 5.24 & 2.27 & 40.38 & 11.90 & E28 & 25.98 & 4.34 & 1.61 & 47.40 & 12.86 \\
\hline 吉 846 Ji 846 & 18.35 & 5.02 & 1.94 & 41.12 & 11.47 & 黄 C Huang C & 32.2 & 4.22 & 1.38 & 47.72 & 12.26 \\
\hline 郑 32 Zheng 32 & 19.01 & 4.99 & 1.93 & 41.59 & 11.77 & 昌 7-2 Chang 7-2 & 18.57 & 5.08 & 1.94 & 41.78 & 12.51 \\
\hline 冀 35 Ji 35 & 17.79 & 5.21 & 1.91 & 41.50 & 12.95 & |掖 515 Ye 515 & 22.22 & 4.84 & 1.77 & 42.84 & 12.03 \\
\hline 9706 & 60.95 & 2.77 & 0.87 & 67.21 & 12.53 & |自 $330 \mathrm{Zi} 330$ & 17.84 & 5.12 & 1.71 & 41.41 & 12.30 \\
\hline 凤直 Fengzhi & 13.18 & 5.52 & 2.12 & 40.07 & 12.96 & 9331 & 21.58 & 4.93 & 1.74 & 43.22 & 12.98 \\
\hline KH5 & 10.66 & 5.39 & 2.18 & 39.99 & 12.29 & $97-21$ & 66.78 & 2.52 & 0.69 & 72.51 & 12.45 \\
\hline 金 54 Jin 54 & 12.58 & 5.46 & 2.30 & 38.60 & 11.37 & |齐 401 Qi 401 & 16.49 & 5.16 & 1.78 & 41.38 & 12.52 \\
\hline VA-91 & 66.49 & 2.50 & 0.74 & 73.03 & 12.22 & Wx303 & 23.72 & 4.85 & 1.59 & 43.08 & 12.38 \\
\hline 冀 815 Ji 815 & 27.8 & 4.04 & 1.68 & 48.59 & 11.49 & WH3143 & 23.92 & 4.72 & 1.65 & 43.08 & 11.45 \\
\hline 338 & 14.44 & 5.33 & 1.94 & 40.24 & 12.29 & H21 & 23.18 & 4.82 & 1.67 & 42.48 & 11.56 \\
\hline $200 B$ & 13.79 & 5.45 & 1.92 & 39.18 & 11.93 & 京 7 黄 Jing 7 Huang & 15.69 & 5.63 & 1.87 & 37.91 & 11.52 \\
\hline 5003 & 33.1 & 4.17 & 1.39 & 47.00 & 11.14 & KW1 & 70.02 & 2.79 & 0.59 & 67.46 & 13.74 \\
\hline Q1982 & 9.91 & 5.67 & 2.37 & 37.82 & 11.63 & K1077 & 16.62 & 5.29 & 1.81 & 40.17 & 12.03 \\
\hline 沈 118 Shen 118 & 21.87 & 4.77 & 1.82 & 43.51 & 12.25 & 掖 488 Ye 488 & 60.91 & 3.10 & 0.80 & 61.29 & 12.91 \\
\hline 7381 & 62.01 & 2.70 & 0.84 & 69.30 & 12.96 & YW02 & 17.9 & 5.48 & 1.87 & 38.66 & 11.61 \\
\hline 3195 & 64.38 & 2.57 & 0.79 & 71.97 & 12.98 & 双 741 Shuang 741 & 17.05 & 5.34 & 1.93 & 39.52 & 11.70 \\
\hline L9801 & 42.33 & 4.14 & 1.28 & 47.89 & 11.88 & YM121 & 17.3 & 5.10 & 1.69 & 40.57 & 11.30 \\
\hline 196 & 24.81 & 4.47 & 1.63 & 45.60 & 12.24 & 7137 & 12.7 & 5.56 & 2.03 & 38.44 & 11.71 \\
\hline 3189 & 33.7 & 3.98 & 1.44 & 50.35 & 12.70 & WA141 & 17.39 & 4.78 & 1.88 & 41.89 & 10.59 \\
\hline JNHZ4 & 23.12 & 4.59 & 1.64 & 44.65 & 12.12 & AY311 & 18.96 & 4.97 & 1.69 & 41.45 & 11.39 \\
\hline 掖 107 Ye 107 & 38.22 & 3.91 & 1.34 & 51.10 & 12.68 & & & & & & \\
\hline
\end{tabular}

MRGLA：成熟期相对绿叶面积; $V_{\text {max }}$ ：相对绿叶面积最大衰减速率; $V_{\mathrm{m}}$ ：相对绿叶面积平均衰减速率; $T_{\max }$ ：相对绿叶面积最大衰 减速率出现的时间; $T_{\mathrm{s}}$ : 植株衰老起始时间。

MRGLA: relative green leaf aera (RGLA) at physiological maturity; $V_{\text {max }}$ : maximum decrease rate of RGLA; $V_{\mathrm{m}}$ : mean decreasing rate of RGLA; $T_{\max }$ : time point when maximum decrease rate occurrs; $T_{\mathrm{s}}$ : the day when plants begin to senescence. 


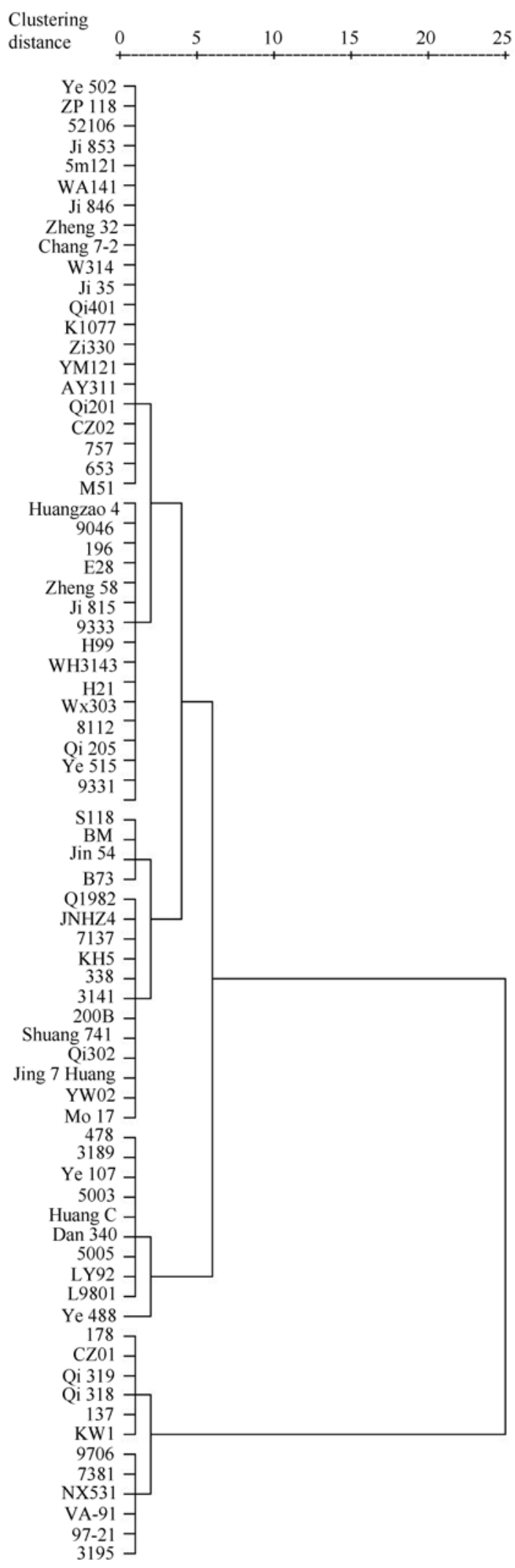

图 2 不同基因型玉米叶片保绿性的聚类分析

Fig. 2 Dendrogram of different maize genotypes based on stay-green traits
型玉米自交系抽丝后叶片保绿度的衰减变化均符合 方程 $y=a \mathrm{e}^{b-c x} /\left(1+\mathrm{e}^{b-c x}\right)$ 。不同基因型间成熟期相对 绿叶面积的变幅差异达极显著水平, 绿叶面积最大 衰减速率和出现最大衰减速率时间差异也达到显著 水平, 而植株衰老起始时间变幅差异不显著, 这说 明叶片衰减速率和衰减持续期对不同基因型玉米自 交系抽丝后叶片保绿度影响较大。

Thomas 和 Smart ${ }^{[9]}$ 根据基因在叶片衰老中的作 用方式和表达时间的不同将保绿作物分为 4 种类型, 这一方法虽然准确, 但却不易获取。Bekavac 等 ${ }^{[20]}$ 、 Willman 等 ${ }^{[35]}$ 依据保绿度把玉米分为保绿型品种和 非保绿型品种, 虽可定量标准保绿度, 却未免单一 和表观化。本试验运用 Hiechical 聚类分析方法, 依 据成熟期相对绿叶面积、叶片衰减速率和衰减持续 期, 可清晰地将所选不同基因型玉米自交系划分为 保绿型和非保绿型。本试验条件下, 保绿型玉米自 交系的界定标准为成熟期相对绿叶面积高于 $60 \%$, 生长期内不出现相对绿叶面积最大衰减速率, 叶片 衰减速率平均为 $0.687 \% \mathrm{~d}^{-1}$; 非保绿型玉米自交系 相对绿叶面积低于 $42.3 \%$, 相对绿叶面积最大衰减 速率出现较早, 平均在抽丝后 $43.02 \mathrm{~d}$, 叶片衰减速 率平均值为 $1.799 \% \mathrm{~d}^{-1}$ 。与非保绿型玉米自交系相 比, 保绿型自交系的成熟期绿叶数和果穗叶片叶绿 素含量分别高 5.0 片和 $2.14 \mathrm{mg} \mathrm{dm}^{-2}$ 。同时, 非保绿 型自交系还可分为植株叶片衰老较快型与植株叶片 衰老较慢型两个亚类。保绿型玉米自交系叶片保绿 度与成熟期的绿叶数、成熟期叶绿素含量、相对绿 叶面积出现最大衰减速率时间、植株衰老时间和成 熟期相对绿叶面积的相关性, 分析表明, 植株保绿 度主要取决于成熟期的绿叶数、成熟期叶绿素含量 和叶片衰减速率。由此认为成熟期的绿叶数、成熟 期叶绿素含量和叶片衰减速率可作为区分玉米保绿 型与非保绿型的关键指标。

在叶龄期内, 延长叶片的功能期, 延缓叶片衰 老能显著提高植株生物产量和籽粒产量 ${ }^{[9,15,36]}$ 。 Waggoner 和 Berger 等 ${ }^{[22]}$ 先后用绿叶面积功能期来 表述玉米叶片保绿性与产量的相关性, 但也有学者 认为玉米保绿性与产量并没有明显的相关 ${ }^{[37-39]}$ 。本 研究发现, 不同基因型玉米自交系抽丝后叶片保绿 性与叶面积持续期、单株产量均呈正相关。保绿型 自交系的叶面积持续期、单株籽粒产量分别比非保绿 型自交系平均高 20.02\% 23.87\%和 50.44\% 59.38\%, 生物产量提高 $21.43 \%$ 28.62\%。保绿型玉米自交系 
表 3 不同自交系植株成熟期绿叶数和叶绿素含量

Table 3 Green leaf number and chlorophyll content of different maize genotypes at maturity

\begin{tabular}{|c|c|c|c|c|c|c|c|c|c|}
\hline & 交系 Inbred line & GLN & CC (\%) & 自交系 Inbred line & GLN & CC (\%) & 自交系 Inbred line & GLN & CC (\%) \\
\hline \multicolumn{10}{|c|}{ 保绿型 Stay-green type } \\
\hline & CZ01 & 9.0 & 4.88 & 137 & 9.0 & 4.46 & 齐 318 Qi 318 & 9.0 & 4.32 \\
\hline & $97-21$ & 8.0 & 4.66 & KW1 & 9.0 & 4.39 & 9706 & 9.0 & 4.28 \\
\hline & 3195 & 9.0 & 4.58 & 齐 319 Qi 319 & 9.0 & 4.36 & VA-91 & 9.0 & 4.20 \\
\hline & 178 & 9.0 & 4.51 & NX531 & 9.0 & 4.35 & 7381 & 8.0 & 4.12 \\
\hline 类型平坅 & 均 Mean of type & 8.8 & 4.43 & & & & & & \\
\hline
\end{tabular}

类型平均 Mean of type $8.8 \quad 4.43$

非保绿型 Non-stay-green type

叶片衰老较快型 Subgroup of with high leaf senescence

\begin{tabular}{|c|c|c|c|c|c|c|c|c|}
\hline CZ02 & 4.0 & 3.68 & 齐 201 Qi 201 & 3.0 & 2.13 & Wh3143 & 3.0 & 1.78 \\
\hline 8112 & 5.0 & 3.58 & 掖 515 Ye 515 & 4.0 & 2.11 & $5 \mathrm{~m} 121$ & 2.0 & 1.76 \\
\hline H99 & 4.0 & 3.28 & Mo17 & 3.0 & 2.06 & 653 & 3.0 & 1.74 \\
\hline 9333 & 5.0 & 3.25 & M51 & 3.0 & 2.06 & 757 & 4.0 & 1.68 \\
\hline 掖 502 Ye 502 & 2.4 & 2.68 & 196 & 5.0 & 2.04 & 齐 401 Qi 401 & 3.0 & 1.68 \\
\hline Ym121 & 2.5 & 2.56 & 9046 & 2.5 & 1.98 & 7137 & 2.0 & 1.67 \\
\hline JNHZ4 & 3.5 & 2.48 & 吉 $846 \mathrm{Ji} 846$ & 3.0 & 1.98 & Wx303 & 3.4 & 1.66 \\
\hline E28 & 5.0 & 2.46 & 齐 302 Qi 302 & 1.8 & 1.91 & H21 & 3.0 & 1.66 \\
\hline 齐 205 Qi 205 & 3.0 & 2.42 & 黄早四 Huangzao 4 & 2.0 & 1.88 & 京 7 黄 Jing 7 Huang & 3.0 & 1.66 \\
\hline WA141 & 3.0 & 2.36 & 冀 35 Ji 35 & 3.0 & 1.88 & KH5 & 3.0 & 1.65 \\
\hline 9331 & 5.0 & 2.35 & AY311 & 3.0 & 1.88 & 200B & 3.0 & 1.65 \\
\hline 郑 32 Zheng 32 & 3.0 & 2.33 & $\mathrm{BM}$ & 3.0 & 1.87 & YW02 & 2.0 & 1.55 \\
\hline 52106 & 2.6 & 2.26 & 凤直 Fengzhi & 3.0 & 1.86 & 金 54 Jin 54 & 3.0 & 1.52 \\
\hline ZP118 & 2.0 & 2.25 & K1077 & 3.0 & 1.85 & 沈 118 Shen 118 & 4.0 & 1.46 \\
\hline 武 314 Wu 314 & 3.0 & 2.18 & 自 $330 \mathrm{Zi} 330$ & 5.0 & 1.84 & 338 & 3.0 & 1.44 \\
\hline 3141 & 3.0 & 2.15 & 双 741 Shuang 741 & 2.4 & 1.82 & Q1982 & 1.5 & 1.28 \\
\hline B73 & 3.0 & 2.14 & 吉 $853 \mathrm{Ji} 853$ & 4.0 & 1.78 & & & \\
\hline
\end{tabular}

亚类平均 Mean of subgroup $3.2 \quad 2.06$

叶片衰老较慢型 Subgroup of with slow leaf senescence

\begin{tabular}{|c|c|c|c|c|c|c|c|c|}
\hline 冀 815 Ji 815 & 5.0 & 3.8 & 5005 & 6.0 & 3.34 & 黄 C Huang C & 8.0 & 2.85 \\
\hline LY92 & 6.0 & 3.64 & 掖 $107 \mathrm{Ye} 107$ & 6.0 & 3.26 & 昌 7-2 Chang 7-2 & 6.0 & 2.42 \\
\hline 478 & 6.0 & 3.59 & 掖 488 Ye 488 & 7.0 & 3.25 & L9801 & 5.0 & 2.16 \\
\hline 丹 340 Dan 340 & 6.0 & 3.44 & 5003 & 8.0 & 3.06 & & & \\
\hline 郑 58 Zheng 58 & 6.0 & 3.42 & 3189 & 6.0 & 2.98 & & & \\
\hline 匀 Mean of subgroup & 6.2 & 3.17 & & & & & & \\
\hline Mean of type & 3.8 & 2.29 & & & & & & \\
\hline
\end{tabular}

GLN: 成熟时绿叶数, 绿叶大于 $1 / 2$ 叶片长的叶片数; CC: 成熟期叶绿素含量。

GLN: green leaf (green part longer than a half of the leaf) number at maturity; CC: content of chlorophyll a and b at maturity.

表 4 叶片保绿性相关性状间的相关系数

Table 4 Correlation coefficient between related to traits leaf stay-green

\begin{tabular}{|c|c|c|c|c|c|c|c|c|c|}
\hline $\begin{array}{l}\text { 性状 } \\
\text { Trait }\end{array}$ & SGD & $L A_{\max }$ & $\begin{array}{c}\text { LA at } \\
\text { maturity }\end{array}$ & $V_{\mathrm{m}}$ & $V_{\max }$ & $T_{\max (\mathrm{V})}$ & $T_{95 \% \mathrm{RGLA}}$ & GLN & $\begin{array}{c}\mathrm{CC} \text { at } \\
\text { maturity }\end{array}$ \\
\hline$L A_{\max }$ & 0.413 & & & & & & & & \\
\hline LA at maturity & $0.984^{* *}$ & 0.537 & & & & & & & \\
\hline$V_{\mathrm{m}}$ & $-0.969^{* *}$ & -0.404 & $-0.952^{* *}$ & & & & & & \\
\hline$V_{\max }$ & $-0.968^{* *}$ & -0.409 & $-0.949^{* *}$ & $0.949^{* *}$ & & & & & \\
\hline$T_{\max (\mathrm{V})}$ & $0.980^{* *}$ & 0.408 & $0.968^{* *}$ & $-0.942^{* *}$ & $-0.969^{* *}$ & & & & \\
\hline$T_{95 \% \text { RGLA }}$ & 0.236 & 0.172 & 0.247 & -0.211 & -0.105 & 0.236 & & & \\
\hline GLN & $0.916^{* *}$ & 0.520 & $0.912^{* *}$ & $-0.896^{* *}$ & $-0.920^{* *}$ & $0.897^{* *}$ & 0.200 & & \\
\hline $\mathrm{CC}$ at maturity & $0.884^{* *}$ & $0.463^{*}$ & $0.882^{* *}$ & $-0.861^{* *}$ & $-0.918^{* *}$ & $0.884^{* *}$ & 0.152 & $0.880^{* *}$ & $0.797^{* *}$ \\
\hline
\end{tabular}

SGD: 保绿度(以生理成熟期的相对绿叶面积表示); $L A_{\max }$ ：最大绿叶面积; LA at maturity：成熟期绿叶面积; $V_{\mathrm{m}}$ : 平均衰减速率; $V_{\max }$ : 最 大衰减速率; $T_{\max (\mathrm{V})}$ ：最大衰减时间; $T_{95 \% \mathrm{RGLA}}$ ：衰减启动时间; GLN: 成熟时绿叶数, 绿叶大于 $1 / 2$ 叶片长的叶片数; CC: 成熟期叶绿素含量。

SGD: stay-green degree (relative green leaf area at physiological maturity); $L A_{\max }$ : maximum area of green leaf; LA at maturity: area of green leaf at maturity; $V_{\mathrm{m}}$ : mean decreasing rate of RGLA; $V_{\max }$ : Maximum reducing rate; $T_{\max (\mathrm{V})}$ : time up to Maximum reducing rate; $T_{95 \% \text { RGLA }}$ time of beginning to senescence; GLN: green leaf (green part longer than a half of the leaf) number at maturity; CC: content of chlorophyll a and $\mathrm{b}$ at maturity.

$r_{0.05}=0.418 ; r_{0.01}=0.745$. 
表 5 不同自交系抽丝后叶面积持续期与单株产量

Table 5 LAD after silking and yield per plant of different maize genotypes

\begin{tabular}{|c|c|c|c|c|c|c|c|c|}
\hline 自交系 Inbred line & LAD & YPP & 自交系 Inbred line & LAD & YPP & 自交系 Inbred line & LAD & YPP \\
\hline \multicolumn{9}{|l|}{ 保绿型 Stay-green type } \\
\hline CZ01 & 24.18 & 96.80 & 齐 319 Qi 319 & 20.17 & 138.60 & 9706 & 16.85 & 75.40 \\
\hline 齐 318 Qi 318 & 21.86 & 143.40 & KW1 & 20.00 & 85.80 & 3195 & 16.60 & 112.50 \\
\hline $97-21$ & 20.87 & 116.80 & 137 & 19.47 & 99.40 & 7381 & 16.06 & 96.80 \\
\hline NY531 & 20.74 & 88.70 & 178 & 19.34 & 92.50 & VA-91 & 15.04 & 68.70 \\
\hline 类型平均 Mean of type & $19.26 \mathrm{a}$ & 101.28 a & & & & & & \\
\hline \multicolumn{9}{|l|}{ 非保绿型 Non-stay-green type } \\
\hline \multicolumn{9}{|c|}{ 叶片衰老较快型 Subgroup of with high leaf senescence } \\
\hline E28 & 16.36 & 76.50 & ZP118 & 11.46 & 57.80 & WA141 & 10.22 & 34.60 \\
\hline 自 $330 \mathrm{Zi} 330$ & 14.05 & 46.80 & 京 7 黄 Jing 7 Huang & 11.43 & 34.80 & 双 741 Shuang 741 & 10.13 & 38.20 \\
\hline CZ02 & 13.64 & 82.40 & 3141 & 11.41 & 41.32 & 齐 302 Qi 302 & 10.07 & 39.10 \\
\hline 8112 & 13.56 & 66.20 & M51 & 11.18 & 54.30 & Yw02 & 10.03 & 36.80 \\
\hline Wx303 & 13.20 & 41.50 & 52106 & 11.11 & 58.30 & 9333 & 10.01 & 59.40 \\
\hline JNHZ4 & 13.18 & 59.50 & 冀 35 Ji35 & 11.04 & 53.20 & B73 & 9.87 & 61.60 \\
\hline 9331 & 13.04 & 48.40 & 齐 401 Qi 401 & 11.00 & 38.70 & 凤直 Fengzhi & 9.83 & 39.80 \\
\hline $\mathrm{H} 21$ & 12.92 & 42.30 & AY311 & 10.93 & 33.20 & 757 & 9.82 & 56.20 \\
\hline 200B & 12.77 & 49.20 & 武 314 Wu 314 & 10.91 & 59.40 & 郑 32 Zheng 32 & 9.61 & 46.80 \\
\hline 9046 & 12.72 & 68.40 & K1077 & 10.79 & 48.20 & $5 \mathrm{~m} 121$ & 9.60 & 34.30 \\
\hline 338 & 12.62 & 47.80 & 齐 205 Qi 205 & 10.78 & 56.00 & 7137 & 9.44 & 36.40 \\
\hline Ym121 & 12.61 & 49.40 & 掖 502 Ye 502 & 10.74 & 55.60 & 吉 846 Ji 846 & 9.31 & 45.40 \\
\hline 196 & 12.21 & 63.60 & Mo17 & 10.73 & 76.40 & 黄早四 Huangzao 4 & 9.31 & 58.60 \\
\hline 掖 515 Ye515 & 12.20 & 56.30 & 沈 118 Shen 118 & 10.54 & 56.20 & KH5 & 8.88 & 34.20 \\
\hline $\mathrm{BM}$ & 12.19 & 76.80 & 653 & 10.36 & 58.40 & 金 54 Jin 54 & 8.27 & 31.10 \\
\hline Wh3143 & 12.07 & 35.90 & 吉 853 Ji 853 & 10.30 & 47.50 & Q1982 & 7.48 & 22.60 \\
\hline 齐 201 Qi 201 & 11.83 & 36.80 & H99 & 10.27 & 45.70 & & & \\
\hline 亚类平均 Mean of subgroup & $11.05 \mathrm{c}$ & $48.37 \mathrm{c}$ & & & & & & \\
\hline \multicolumn{9}{|c|}{ 叶片衰老较慢型 Subgroup of with slow leaf senescence } \\
\hline 黄 C Huang C & 17.41 & 78.60 & 郑 58 Zheng 58 & 13.91 & 90.10 & L9801 & 12.83 & 58.90 \\
\hline 5005 & 15.94 & 65.50 & 掖 107 Ye 107 & 13.86 & 53.60 & 昌 7-2 Chang 7-2 & 11.64 & 54.80 \\
\hline 5003 & 15.84 & 94.20 & 478 & 13.74 & 69.80 & 冀 815 Ji 815 & 11.41 & 56.30 \\
\hline 丹 340 Dan 340 & 15.66 & 75.40 & 3189 & 13.72 & 67.10 & & & \\
\hline 掖 488 Ye 488 & 14.18 & 69.60 & LY92 & 13.71 & 96.60 & & & \\
\hline 亚类平均 Mean of subgroup & $13.75 \mathrm{~b}$ & $70.78 \mathrm{~b}$ & & & & & & \\
\hline 类型平均 Mean of type & $11.61 \mathrm{c}$ & 52.99 с & & & & & & \\
\hline
\end{tabular}

LAD: 叶面积持续期 $\left(\mathrm{m}^{2} \mathrm{~d}\right)$, YPP: 单株产量 $(\mathrm{g})$ 。平均值后不同小写字母分别表示类型间差异达到 0.05 显著水平。

LAD: leaf area duration after silking $\left(\mathrm{m}^{2} \mathrm{~d}\right)$; YPP: yield per plant (g). Different lowercase letters indicate significant difference between types or subgroups at $P<0.05$.

$d f=73, t_{\mathrm{LAD}}=11.062, p_{\mathrm{LAD}}=0.000 ; t_{\mathrm{YPP}}=8.587, p_{\mathrm{YPP}}=0.000$. 

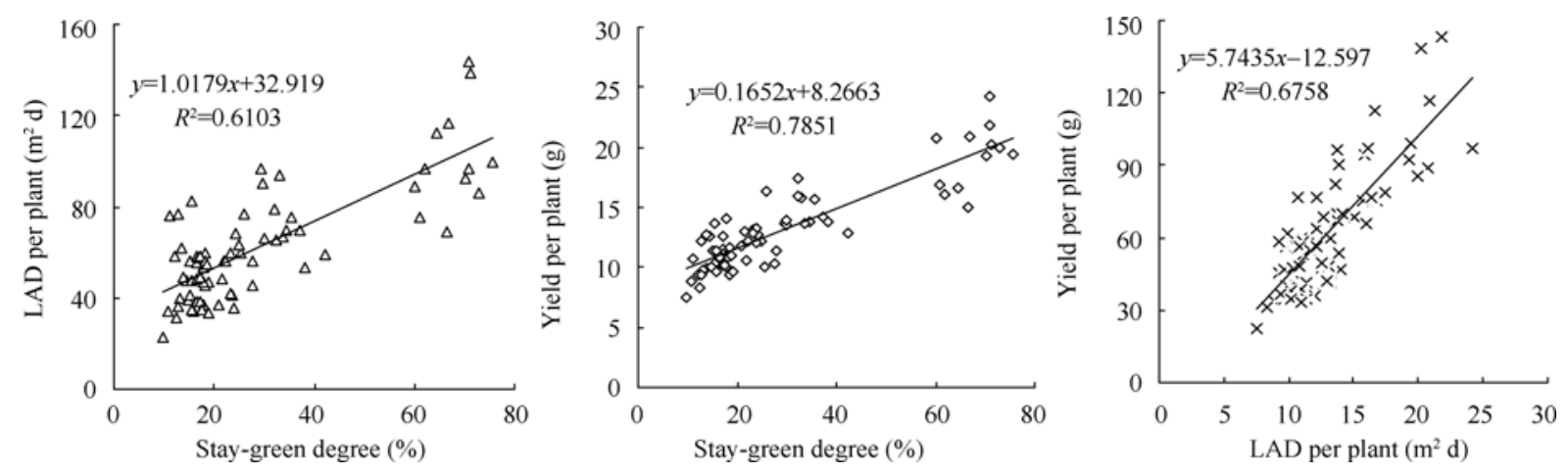

图 3 叶片保绿度与 LAD、单株产量间的相关

Fig. 3 Correlation among leaf stay-green degree, LAD, and yield per plant

的叶面积持续期较长, 利于延长叶片的光合作用时 间，在籽粒灌浆期积累更多的干物质，从而提高产量。

\section{4 结论}

不同基因型玉米自交系抽丝后叶片保绿度的衰 减进程不一, 变化趋势均符合方程 $y=a \mathrm{e}^{b-c x} /(1+$ $\mathrm{e}^{b-c x}$ 。室米成熟期的绿叶数、成熟期叶绿素含量和 叶片衰减速率可作为判断玉米保绿性能的关键指标, 12 个保绿型自交系在成熟期的绿叶数可达 8 9 叶, 叶绿素含量平均 $2.06 \mathrm{mg} \mathrm{dm}^{-2}$, 叶片衰减速率平均 $1.89 \% \mathrm{~d}^{-1}$ 。玉米保绿度与产量呈显著正相关, 由于 具有较长的光合作用时间和较高的群体光合速率, 保绿型自交系单株产量(101.28 g)明显高于非保绿型 (52.99 g)。

\section{References}

[1] Pan R-C(潘瑞炽). Plant Physiology (植物生理学). Beijing: Higher Education Press, 2004 (in Chinese)

[2] Thomas H, Howarth C J. Five ways to stay green. J Exp Bot, 2000, 51: 329-337

[3] Subudhi P K, Rosenow D T, Nguyen H T. Quantitative trait loci for the stay-green trait in sorghum (Sorghum bicolor L. Moench): Consistency across genetic backgrounds and environments. Theor Appl Genet, 2000, 101: 733-741

[4] Haussmann B I G, Mahalakshmi V, Reddy B V S. QTL mapping of stay-green in two sorghum recombinant inbred populations. Theor Appl Genet, 2002, 106: 133-142

[5] Tollenaar M, Daynard T B. Leaf senescence in short-season maize hybrids. Can J Plant Sci, 1978, 58: 869-874

[6] Ceppi D, Sala M, Gentinetta E. Genotype-dependent leaf senescence in maize: 1 . Inheritance and effects of pollination-prevention. Plant Physiol, 1987, 85: 720-725

[7] Craft-brandner S J, Below F E, Wittenbach V A. Effect of ear removal on $\mathrm{CO}_{2}$ exchange and activties of ribulose bisphate earboxylase and phosphoenlpyruvate carboxylase of maize hybrids and inbred lines. Plant Physiol, 1987, 84: 261-265
[8] Phillips D A, Pierce R O, Edie S A, Foster K A, Knowles P F. Delay leaf senescence in soybean. Crop Sci, 1984, 24: 518-522

[9] Thomas H, Smart C M. Crops that stay green. Ann Appl Biol, 1993, 123: 193-219

[10] Xu W W, Rosenow D T, Nguyen H T. Stay-green trait in grain sorghum: relationship between visual rating and leaf chlorophyll concentration. Plant Breed, 2000, 119: 365-367

[11] Borrell A K, Hammer G H. Does maintaining green leaf area in sorghum improve yield under drought? II. Dry matter production and yield. Crop Sci, 2000, 40: 1037-1048

[12] Gentinetta E, Ceppi D, Lepori C, Perico G, Motto M, Salamini F. A major gene for delayed senescence in maize: Pattern of photosynthates accumulation and inheritance. Plant Breed, 1986, 97: 193-203

[13] Duncan R R. The association of plant senescence with root and stalk diseases in sorghum. In: Mughogho L K, Rosenberg G, eds. Sorghum Root and Stalk Rots: A Critical Review. Proceedings of the Consultative Group Discussion on Research Needs and Strategies for Control of Sorghum Root and Stalk Rot Diseases. Bellagio, Italy \& Patancheru, India: ICRISAT, 1984. pp 99-110

[14] Guo Q-F(郭庆法), Wang Q-C(王庆成), Wang L-M(汪黎明). Maize Cultivation in China (中国玉米栽培学). Shanghai: Shanghai Scientific and Technical Publishers, 2004. pp 32-34 (in Chinese)

[15] Dong S-T(董树亭), Wang K-J(王空军), Hu C-H(胡昌浩). Development of canopy apparent photosynthesis among maize varieties from different eras. Acta Agron Sin (作物学报), 2000, 26(2): 200-204 (in Chinese with English abstract)

[16] Duvick D N. Genetic rates of grain in hybrid maize yields during the past 40 years. Maydica, 1997, 23: 187-196

[17] Russell W A. Registration of B93 and B94 inbred lines of maize. Crop Sci, 1991, 31: 247-248

[18] Choi K J, Chin M S, Park K Y, Kim S L, Chung T W, Lee H S. Segregation of stay-green characters in an $\mathrm{F}_{2}$ population. Maize Genetics Cooperation Newsl, 1995, 69: 123

[19] Chfistens L E, Below F E, Hagreman R H. The effect of ear removal on sevwscence and metabolism of maize. Plant Physiol, 1981, 68: 1180-1185 
[20] Bekavac G. Path analysis of stay-green trait in maize. Cereal Res Commun, 1998, 26: 161-167

[21] Walulu R S, Darrell T R, Wester D B, Nguyen H T. Inheritance of the stay green trait in sorghum. Crop Sci, 1994, 34: 970-972

[22] Waggoner P E, Berger R D. Defoliation, disease, and growth. Phytioathology, 1987, 77: 393-398

[23] Wang J-G(王建国), Du G-J(杜桂娟), Cai J-X(蔡纪新), Zhang B-S(张宝石). Relationship between stay-green and other agronomic traits in maize. Rain Fed Crops (杂粮作物), 2003, 23(6): 336-339 (in Chinese with English abstract)

[24] Craft-Brandner S J, Below F E, Wittenbach V A, Harper J E, Hageman R H. Differential senescence of maize hybrids following ear removal: II. Selected leaf. Plant Physiol, 1984, 74: 368-373

[25] Yang J-P(杨俊品), Rong T-Z(荣廷昭), Xiang D-Q(向道权), Tang H-T(唐海涛), Huang L-J(黄烈健), Dai J-R(戴景瑞). QTL mapping of quantitative traits in maize. Acta Agron Sin (作物学报), 2005, 31(2): 188-196 (in Chinese with English abstract)

[26] Liu K-C(刘开昌), Wang Q-C(王庆成), Zhang H-S(张海松), Feng K(冯凯). Advance in research of physiological mechanism and genetic traits of stay-green of maize. Shandong Agric Sci (山 东农业科学), 2003, (2): 48-51(in Chinese with English abstract)

[27] Zheng H-J(郑洪建), Shen X-F(沈雪芳), Wu A-Z(吴爱忠). The research advance in inheritance of stay-green traits of maize leaf blades. Acta Agric Shanghai (上海农业学报), 2007, 23(4): 9094 (in Chinese with English abstract)

[28] Zhu Z-P(朱治平). Laboratory Manual of Plant Physiology (植物 生理学实验手册). Shanghai: Shanghai Scientific and Technical Publishers, 1985 (in Chinese)

[29] van Oosterom E J, Jayachandran R, Bidinger F R. Diallel analysis of the stay green trait and its components in sorghum. Crop Sci, 1996, 36: 549-555

[30] Nguyen H T, Xu W W, Rosenow D T, Mullet J E, McIntyre L. Use of biotechnology in sorghum drought resistance breeding. In: Proceedings of the International Conference on Genetic Im- provement of Sorghum and Pear Millet, Lubbock, Texas, USA, 1996. pp 412-424

[31] Crasta O R, Xu W W, Rosenow D T, Mullet J E, Nguyen H T. Mapping of post-flowering drought resistance traits in grain sorghum: Association of QTLs influencing senescence and maturity. Mol Gen Genet, 1999, 262: 579-588

[32] Wan C, Xu W W, Sosebee R E, Machado S, Archer T. Hydraulic lift in drought-tolerant and -susceptible maize hybrids. Plant \& Soil, 2000, 219: 117-126

[33] Thomas H, Morgan W G, Thomas A M, Ougham H J. Expression of the stay-green character introgressed into Lolium temulentum Ceres from a senescence mutant of Festuca pratensis. Theor Appl Genet, 1999, 99: 92-99

[34] Smart C M, Hosken S E, Thomas H, Greaves J A, Blair B G, Schuch W. The timing of maize leaf senescence and characterisation of senescence-related cDNAs. Physiol Plant, 1995, 93: 673-682

[35] Willman M R, Below F E, Lambert R J, Howey A E, Mies D W. Plant traits related to productivity of maize: II. Development of multiple trait models. Crop Sci, 1987, 27: 1122-1126

[36] Dong S-T(董树亭), Gao R-Q(高荣岐), Hu C-H(胡昌浩), Wang Q-Y(王群瑛), Wang K-J(王空军). Study of canopy photosynthesis property and high yield potential after anthesis in maize. Acta Agron Sin (作物学报), 1997, 23(3): 318-325 (in Chinese with English abstract)

[37] Wada Y, Wada G. Varietal difference in leaf senescence during ripening period of advanced indica rice. Jpn J Crop Sci, 1991, 60: 529-536

[38] Bolanos J, Edmeades G O. The importance of the anthesis-silking interval in breeding for drought tolerance in tropical maize. Field Crops Res, 1996, 48: 65-80

[39] Jiang G H, He Y Q, Xu C G, Li X H, Zhang Q. The genetic basis of stay-green in rice analyzed in a population of doubled haploid lines derived from an indica by japonica cross. Theor Appl Genet, 2004, 108: 688-698

\section{欢迎订阅 2010 年《园艺学报》}

《园艺学报》是中国园艺学会主办的学术期刊, 创刊于 1962 年, 刊载有关果树、蔬菜、观赏植物、茶 及药用植物等方面的学术论文、研究简报、专题文献综述、问题与讨论、新技术新品种以及园艺研究动态 与信息等, 适合园艺科研人员、大专院校师生及农业技术推广部门专业技术人员阅读参考。

《园艺学报》是中文核心期刊，被中国科学引文数据库等多家重要数据库收录。2005 年荣获第三届国 家期刊奖, 2008 年获中国科技信息所“ 中国精品科技期刊”称号及武汉大学中国科学评价研究中心“中国权 威学术期刊” 称号。据 2008 年《中国科技期刊引证报告》 (扩刊版) 登载, 2007 年《园艺学报》总被引频次 4213 次, 影响因子 1.323。

《园艺学报》为月刊，每月 25 日出版。2010 年每期定价 40.00 元，全年 480.00 元。国内外公开发行，全 国各地邮局办理订阅，国内邮发代号 82-471，国外发行由中国国际图书贸易总公司承办，代号 M448。漏订 者可直接寄款至本编辑部订购。

编辑部地址: 北京市海淀区中关村南大街 12 号 中国农业科学院蔬菜花卉研究所《园艺学报》编辑部; 邮政编码: 100081; 电话: (010) 82109523。E-mail:yuanyixuebao@126.com; 网址: http://www.ahs.ac.cn。 